\title{
Un estudio ecológico sobre tuberculosis en un municipio de Cuba
}

\author{
An ecologic study on tuberculosis \\ in a Cuban municipality
}

Ivette Molina Serpa 1

Cándido López Pardo 2

Ricardo Alonso Hernández 3

\footnotetext{
1 Subdirección de Epidemiología, Instituto de Medicina Tropical "Pedro Kourí". Apartado Postal 601 Marianao 13, Ciudad de La Habana, Cuba. ims@ipk.sld.cu

2 Facultad de Economía, Universidad de La Habana. Calle L \# 353/21 y 23, Vedado, Plaza de la Revolución, Apartado Postal 10400, Ciudad de La Habana, Cuba.

3 Unidad de Análisis y Tendencias de Salud. Calle 116 esq. 49, Apartado Postal 11500, Marianao, Ciudad de La Habana, Cuba.
}

\begin{abstract}
An ecological study analyzes tuberculosis incidence in the municipality of Marianao, Havana City Province, Cuba. The study characterizes tuberculosis incidence, identifies spatial distribution patterns, and relates tuberculosis incidence rates to socioeconomic factors. The spatial units are the 29 neighborhoods in the municipality of Marianao. A pattern of neighborhoods with high rates located in the central region of the municipality is identified. Incidence rates are directly (and significantly) related to the percentage of families presenting dysfunctional problems and the percentage of the population with certain levels of overcrowding and inversely related to population density, while not significantly associated with level of schooling or prevailing housing conditions.
\end{abstract}

Key words Tuberculosis; Socioeconomic Factors; Incidence

Resumen Se aplica un estudio ecológico para analizar la incidencia de tuberculosis en el municipio Marianao de la provincia Ciudad de La Habana de la República de Cuba en el período 1995-2000. Se realiza una caracterización de tal incidencia, se identifican patrones de distribución espacial y se determina la relación existente entre los niveles de incidencia de tuberculosis y factores del medio ambiente socioeconómico. La unidad espacial considerada son los 29 barrios del municipio Marianao. Entre otros resultados se identifica un patrón de barrios con tasas altas en la región central del municipio. Las tasas de incidencia se hallan significativamente asociadas de forma directa con el porcentaje de familias con problemas disfuncionales y con el porcentaje de población con determinados niveles de hacinamiento, y de manera inversa con la densidad poblacional, y no se encuentran significativamente asociadas ni con los niveles de educación, ni con el estado de la vivienda predominantes en el barrio.

Palabras-clave Tuberculosis; Factores Socioeconómicos; Incidencia 


\section{Antecedentes}

En los últimos años se ha manifestado un creciente interés en el desarrollo de métodos para el análisis espacial de eventos en salud (Bailey, 2001; Cámara \& Vieira, 2001; Fonseca, 1996; Werneck, 1997), y se han realizado diversas investigaciones empleando estos métodos para identificar áreas donde residen grupos de población que requieren una prioridad de intervención relacionada con la incidencia de diversas enfermedades, entre ellas la tuberculosis. Por ejemplo, en investigaciones ejecutadas en ciudades del estado de Pernambuco (Olinda y Recife), Brasil, relacionadas con la incidencia de tuberculosis y lepra se emplearon diversos métodos de análisis y diferentes escalas espaciales. En el estudio de la ciudad de Olinda (Vieira, 2000) se calcula el Índice de Deprivación Social y se emplea el análisis de componentes principales y el análisis de cluster para estratificar el espacio urbano a nivel censal y determinar la asociación entre el gradiente de riesgo de tuberculosis y la incidencia media de la enfermedad. A su vez, en el estudio realizado en la ciudad de Recife (Vieira, 2001), se analiza la distribución espacial de lepra a nivel de barrio empleando el método bayesiano empírico para la suavización de las tasas y se identifican áreas de alto riesgo asociadas a malas condiciones de vida. Otros trabajos (Bishai et al., 1998) proponen la identificación de patrones espaciales de transmisión de tuberculosis mediante la identificación de focos geográficos basados en los contactos epidemiológicos de los casos y su relación con el nivel socioeconómico del barrio.

En Cuba, desde 1962 se estableció el Programa Nacional de Control de Tuberculosis, el cual se organiza en todos los niveles territoriales: nacional, provincial, municipal y de área de salud y se ejecuta desde el nivel local con normas que se extienden a todas las unidades de salud (MINSAP, 1999a). No obstante, no existen estudios anteriores que relacionen la incidencia de tuberculosis con factores socioeconómicos desde un enfoque espacial, por lo que se realiza este trabajo tomando un municipio de la capital del país.

\section{Unidades espaciales consideradas y población objeto de estudio}

Marianao constituye uno de los 15 municipios de la provincia Ciudad de La Habana (Figura 1), con una población de 138.504 habitantes en el año 2000, lo que representaba el 6,33\% de la provincia (Oficina Nacional de Estadísticas, 2000).

Según la concepción de espacio como un conjunto de relaciones y de formas que se pre-

Figura 1

Ubicación del área de estudio.

Provincia Ciudad de La Habana
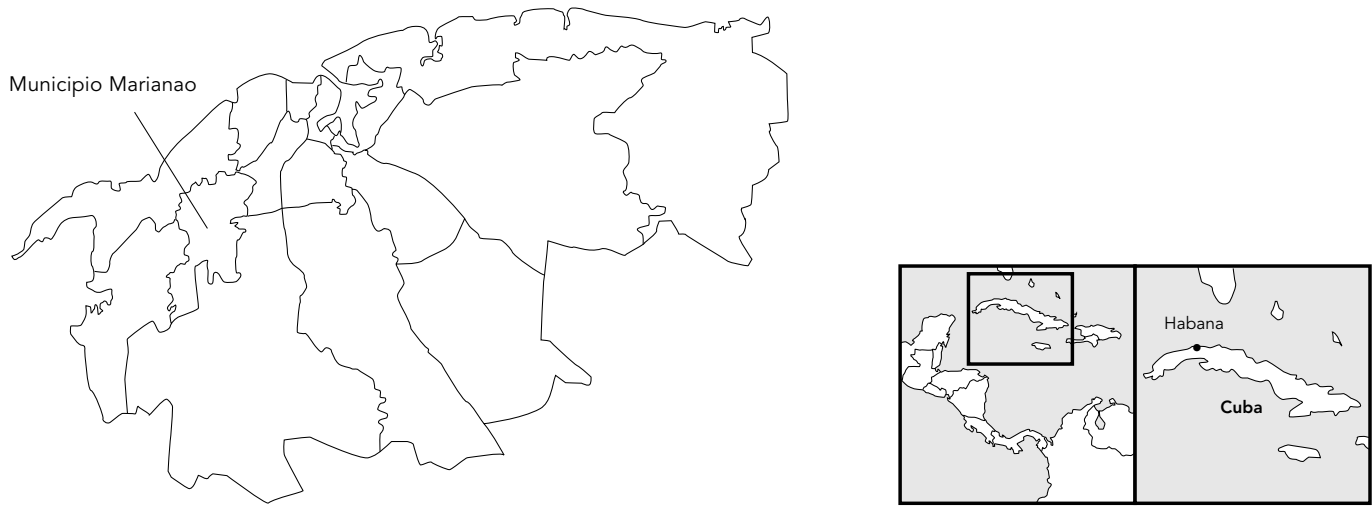
sentan como testimonio de una historia escrita de los procesos del pasado y del presente, (Santos, 1994), la unidad espacial considerada en este estudio son los 29 barrios social e históricamente construidos del municipio Marianao. La población objeto de estudio son todos los casos reportados de tuberculosis pulmonar o extrapulmonar residentes en los barrios del municipio Marianao en los años 1995 al 2000, período en que se observa un alza de la incidencia de tuberculosis en el país en la década de los 90 (MINSAP, 1998, 1999b, 2000, 2001), y en particular en el municipio objeto de estudio.

\section{Objetivos del trabajo}

1) Caracterizar la incidencia de tuberculosis en el período 1995-2000 en el municipio Marianao de la provincia Ciudad de La Habana de la República de Cuba.

2) Identificar el patrón de distribución espacial de la incidencia de tuberculosis en el período de estudio considerado.

3) Determinar la relación existente entre los niveles de incidencia de la tuberculosis y diferentes categorías de factores del medio ambiente socioeconómico.

\section{Procedimientos}

La identificación de las relaciones que pueden darse entre las diversas categorías de factores socioeconómicos y la incidencia de tuberculosis se realiza a través de un enfoque ecológico. Según Silva (1996) - quien reivindica el uso de este enfoque - los estudios ecológicos han ido perdiendo presencia en la investigación epidemiológica contemporánea debido en parte al temor que despierta la falacia ecológica y en parte a prejuicios, de ellos el más arraigado y pernicioso, la convicción de que las variables medidas a nivel de grupo no representan agentes causales de enfermedad. A su vez, señalan Kunts \& Mackenbach (s.d.), los estudios ecológicos permiten identificar la presencia de desigualdades en salud y pudiera ser la vía fundamental de descubrir la existencia de desigualdades en salud en algunos países. La comparación entre áreas, exponen estos autores, puede proporcionar información relevante para el desarrollo de políticas de salud locales al identificar con nitidez áreas con excesos de problemas de salud.

Los indicadores socioeconómicos considerados para cada barrio son:
1) Nivel de escolaridad predominante: primaria, secundaria, preuniversitario y universitario.

2) Porcentaje de familias disfuncionales (con problemas socioeconómicos, psicológicos y/o de higiene ambiental): aquellas que presentan problemas en el equilibrio armónico de su salud y sus condiciones socioeconómicas y culturales (Álvarez-Sintes, 2001).

3) Estado técnico de la vivienda predominante: bueno, regular o malo. Esta clasificación se obtiene después de valorar por puntuación el estado de los elementos componentes de la vivienda, (Instituto Nacional de la Vivienda, 1990).

4) Porcentaje de población residiendo en diferentes niveles de hacinamiento: bajo (hasta 3 personas por habitación para dormir), medio (4 o 5 personas por habitación) o alto (más de 5 personas por habitación).

5) Densidad poblacional.

Para conformar la base de datos se utilizó el Sistema de Información Geográfica (SIG) MapInfo 5.0 constituyendo grupos con diferentes informaciones brindadas por la Dirección Municipal de Arquitectura y Urbanismo (DAU) y la Dirección Municipal de Salud de Marianao.

La capa barrios, de tipo región, representa el límite por barrios y contiene la información de su área en $\mathrm{m}^{2}$. La capa manzanas es de tipo región y contiene la información del estado técnico de la vivienda y del número de habitantes en 1997. La capa consultorios es de tipo región, representa el área que atiende cada Consultorio Médico de la Familia y contiene la información del número de habitantes total, número total de familias, número de familias disfuncionales, número de habitantes según nivel de escolaridad y número de habitantes residiendo en las tres categorías de hacinamiento consideradas.

La capa casos es de tipo puntos, representa la localización de cada caso de tuberculosis en el período 1995-2000 en su lugar de residencia y contiene la información de su dirección particular y la fecha de diagnóstico.

Para obtener los indicadores: nivel de escolaridad predominante, porcentaje de familias disfuncionales, porcentaje de población residiendo en diferentes niveles de hacinamiento y densidad poblacional se superponen las capas consultorios y barrios en el SIG y se realiza la suma de los valores de la información que contienen los consultorios que se encuentran dentro de cada barrio.

Se calcula para cada barrio:

- El porcentaje de habitantes con nivel de escolaridad de primaria, secundaria, preuniver- 
sitario y universitario del total de habitantes y se caracteriza cada barrio con el nivel más frecuente.

- El porcentaje de familias disfuncionales del total de familias.

- El porcentaje de habitantes residiendo en condiciones de hacinamiento alto, medio y bajo, del total de habitantes.

- La densidad poblacional como el cociente entre el número de habitantes y el área de cada barrio en $\mathrm{m}^{2}$.

Para obtener el indicador estado de la vivienda predominante, se superponen las capas manzanas y barrios. Se realiza la suma del número de viviendas total y del número de viviendas según estado técnico en las manzanas que se encuentran dentro de cada barrio. Se calcula para cada barrio el porcentaje de viviendas en bueno, regular y mal estado técnico y se caracteriza el barrio según el estado técnico más frecuente.

Para obtener las tasas observadas por barrio, se superponen las capas casos y barrios y se calcula el número de casos por barrio. Se superponen las capas manzanas y barrios para hallar la población por barrio en 1997. La tasa para cada barrio se obtiene como el cociente entre el número de casos acumulados en los años 1995 y 2000 y seis veces la población del barrio registrada en 1997, multiplicado este cociente por 1.000 .

Para el cumplimiento del primer objetivo se compara la tasa media registrada a nivel municipal en el período considerado con las tasas a nivel nacional y provincial y se describen valores centrales de las tasas observadas a nivel de barrio. La tasa para cada barrio se obtiene como el cociente entre el número de casos acumulados en los años 1995 y 2000 y seis veces la población del barrio registrada en 1997, multiplicado este cociente por 1.000. Para cumplir el segundo objetivo las tasas observadas fueron transformadas en tasas ajustadas mediante la aplicación de un modelo bayesiano empírico (Vieira, 2001). El abordaje bayesiano, del cual el método empleado en este estudio es una de las formas de abordaje, se recomienda para el análisis espacial de enfermedades cuando como en el caso que nos ocupa - las unidades geográficas tienen pequeñas poblaciones en riesgo (Martins, 1998). Para cumplimentar el tercer objetivo se emplearon los siguientes procedimientos de acuerdo al análisis realizado: prueba de Wilcoxon-Mann-Whitney dócima de Kruskal-Wallis, y prueba $\chi^{2}$ de significación de la asociación (Bradley, 1968; Kanji, 1993; Mosteller \& Rourke, 1973; Siegel, 1956; Steel \& Torrie, 1960).
La prueba de Wilcoxon-Mann-Whitney se aplica a 2 muestras independientes con datos en escala de intervalo o de razón y dócima, la hipótesis nula de que las muestras provienen de poblaciones que estrictamente no difieren en sus valores centrales (prueba de 2 colas), o que los valores centrales de una población son mayores o iguales, o menores o iguales, que los de la otra (pruebas de 1 cola). Constituye la versión no paramétrica de la prueba $z$ (aplicable cuando las varianzas poblacionales son conocidas), de la prueba $t$ (cuando se desconocen las varianzas poblacionales pero se asume, o se demuestra, homocedasticidad) y de la prueba de Behrens-Fisher (cuando las varianzas poblacionales son desconocidas y no estamos en presencia de homocedasticidad).

La dócima de Kruskal-Wallis puede aplicarse a $k \geq 3$ muestras independientes cuando los datos se encuentran en una escala de intervalo o de razón. Pone a prueba la hipótesis nula de que las $k$ poblaciones subyacentes se encuentran igualmente distribuidas. Constituye el equivalente no paramétrico de, entre otras pruebas, la dócima $\chi^{2}$ (cuando se conocen las $k$ varianzas poblacionales) y del Análisis de Varianza de un Criterio de Clasificación (en el caso de que las variantes sean desconocidas pero existe homocedasticidad).

La prueba $\chi^{2}$ de significación de la asociación es una dócima de hipótesis no paramétrica que, a los efectos del presente análisis, pone a prueba la hipótesis nula de que existe independencia entre las dos variables consideradas. Para esta prueba, de amplia utilización, las hipótesis pueden expresarse de maneras diferentes.

\section{Resultados}

La tasa media observada de incidencia de tuberculosis en el período 1995-2000 en el municipio de Marianao es de 0,224 por 1.000 habitantes en contraste con la tasa media anual del país en el propio período que fue de 0,118 y la tasa media anual para las mismas fechas en la provincia de Ciudad de La Habana de 0,152 por 1.000 habitantes. Es decir, en la etapa 19952000, el riesgo relativo de un habitante del municipio de Marianao de contraer la tuberculosis fue casi 2 veces mayor que el de un habitante del país en su totalidad, y de 1,5 veces mayor que el de un habitante de la provincia Ciudad de La Habana.

Las tasas ajustadas a nivel de barrio para el período objeto de análisis oscilaron entre 0,1721 por 1.000 habitantes en el barrio Coco Solo y 
0,3055 en el barrio de Columbia. De los 29 barrios, 12 presentaron tasas ajustadas superior a la media municipal de 0,2205 por 1.000 habitantes.

Se identifica un agrupamiento significativo de barrios con tasas altas (superiores a la media del municipio) concentrados en la región central del municipio (Figura 2). Los barrios que conforman este agrupamiento y las tasas (por 1.000 habitantes) que registran son los siguientes: Columbia $(0,305)$, Rivero $(0,235)$, Padre Zamora $(0,244)$, Machín $(0,242)$, Los Quemados $(0,232)$, Buen Retiro $(0,266)$, Libertad $(0,237)$, Pogolotti $(0,262)$ y Loma de Llave $(0,267)$.

En relación a las diferencias entre las tasas según porcentaje de familias disfuncionales, se halla que aquellos barrios con un $12 \%$ o más de familias disfuncionales tienen significativamente una mayor tasa de incidencia de tuberculosis $(z=-1,798 ; p=0,0361)$. En los barrios con este porcentaje inferiores al $12 \%$ la tasa media es de 0,2147 por 1.000 habitantes y un valor mediano de 0,2069 , en tanto en aquellos barrios con porcentajes iguales o superiores al $12 \%$ el promedio de la tasa ajustada es de 0,2302 y la mediana de 0,2210 .

En lo que respecta a las diferencias entre las tasas ajustadas de acuerdo a niveles de población viviendo con diversos grados de hacinamiento, se encuentra que los barrios con un $90 \%$ o más de población con hacinamiento medio o alto tienen significativamente una mayor tasa de incidencia de tuberculosis $(\mathrm{z}=-2,340 ; \mathrm{p}=$ 0,0096). En los barrios con tal porcentaje menores del $90 \%$ la tasa promedio es de 0,2112 por 1.000 habitantes y un valor de la mediana igual a 0,2096 , mientras en los barrios con porcentaje de población viviendo con medio o alto hacinamiento igual o mayor al $90 \%$ la tasa media ajustada es de 0,2384 y la mediana de 0,2314 .

En lo que concierne a las diferencias de tasas según niveles de densidad poblacional, se encuentra que aquellos barrios con densidad poblacional inferior a 0,025 habitantes por $\mathrm{m}^{2}$ tienen significativamente mayor tasa de incidencia acumulada de tuberculosis que los barrios con densidad poblacional igual o mayor que $0,025(z=2,593 ; p=0,0048)$. En el primer grupo la tasa media es de 0,2253 y la mediana de 0,2173 , mientras que en los barrios con densidad poblacional igual o superior a 0,025 el promedio de la tasa de incidencia es 0,191 y un valor mediano igual a 0,194 .

En el Figura 3 se muestran los factores de riesgo identificados por barrio a partir del estudio ecológico realizado (barrios con un $12 \%$ o más de familias disfuncionales, con un $90 \%$ o
Figura 2

Tasas de incidencia de tuberculosis ajustadas por barrio.

Municipio Marianao, Cuba, 1995-2000.

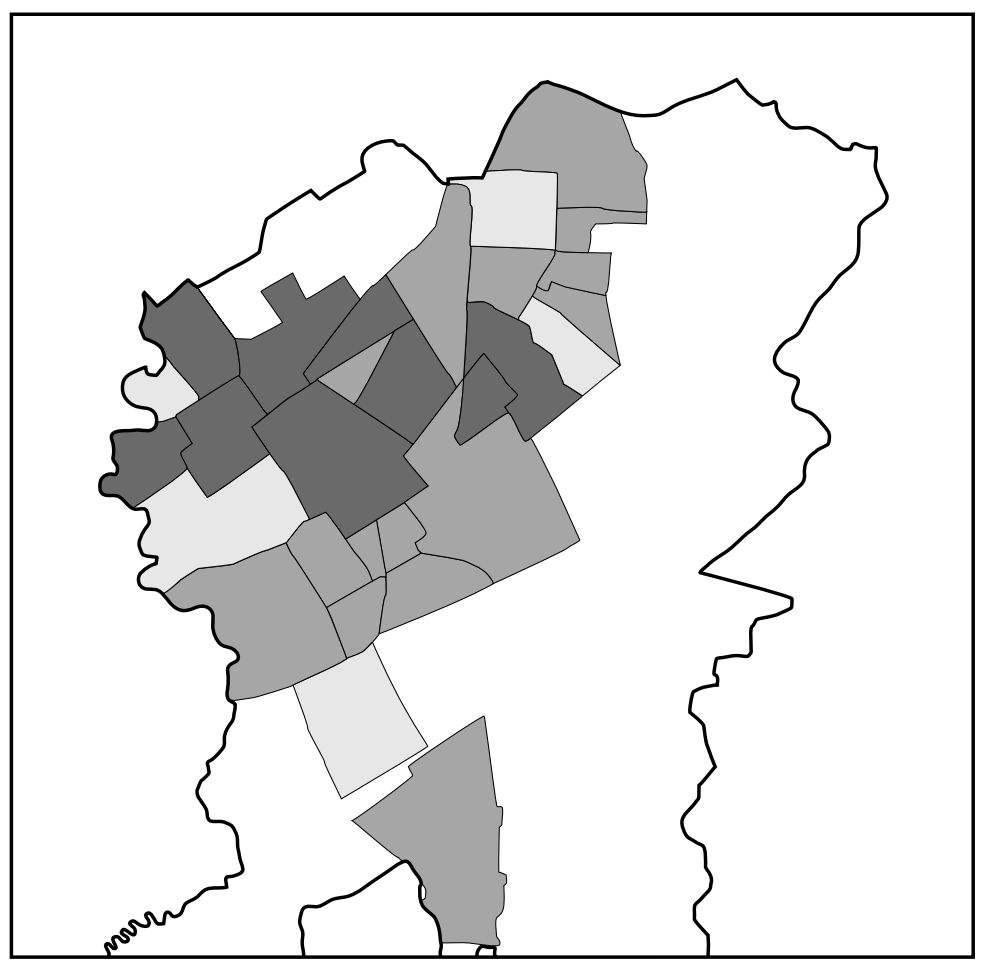

más de población con hacinamiento medio o alto y con una densidad poblacional menor a 0,025 habitantes por $\mathrm{m}^{2}$ ).

Se halla, por otra parte, un incremento de las tasas de incidencia media de tuberculosis según el número de factores de riesgo que presenta el barrio. En aquellos sin ninguno de estos factores de riesgo la tasa promedio es de 0,198 por 1.000 habitantes, con un factor de riesgo la tasa media es de 0,202 , con dos factores de riesgo es de 0,230 como promedio, y con los tres factores de riesgo presentes la tasa promedio de incidencia de tuberculosis es de 0,254 
Factores de riesgo identificados a nivel de barrio.

Municipio Marianao, Cuba, 1995-2000.

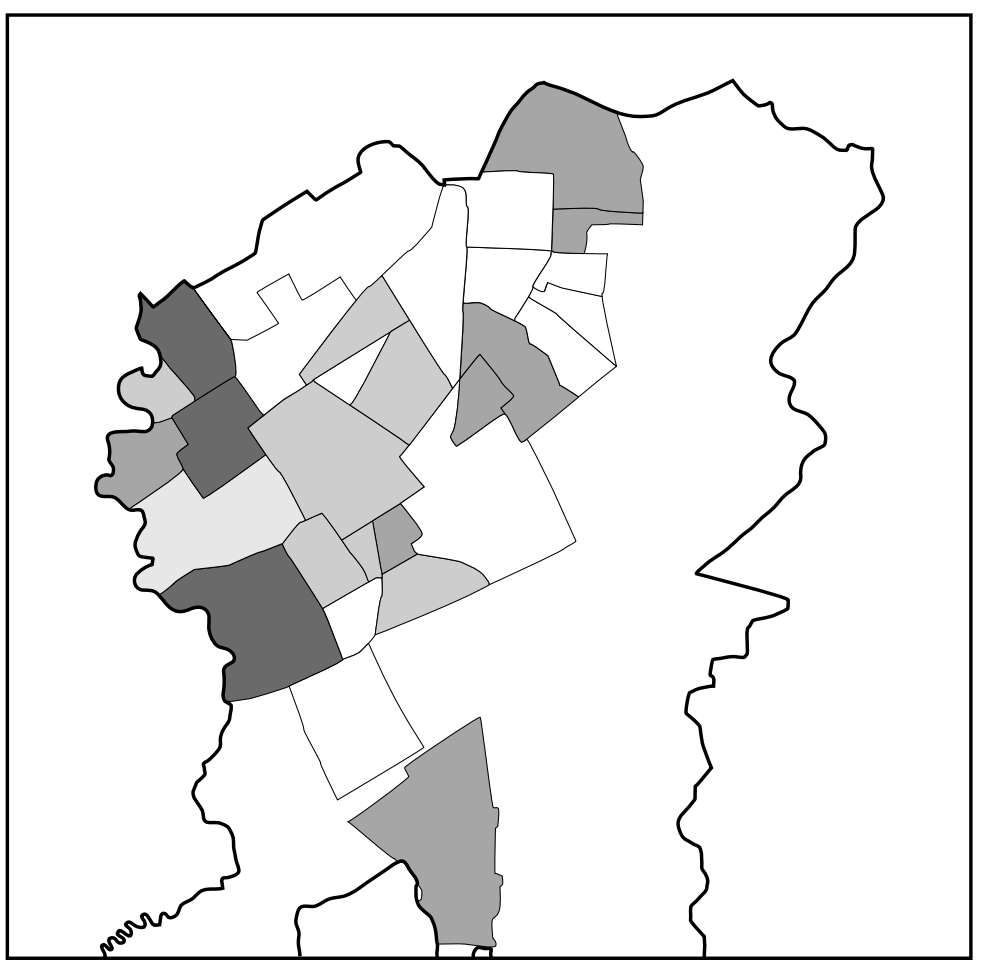

Presencia del factor 1

Presencia del factor 2

Presencia de los factores 1 y 2

Presencia de los factores 1 y 3

Presencia de los tres factores de riesgo

— Limite municipal

_ Limite de barrio

Factores de riesgo identificados a nivel de barrio

1) Densidad poblacional $<0,025$ habitantes $/ \mathrm{m}^{2}$.

2) Presencia del $12 \%$ y más de familias disfuncionales

3) Presencia del $90 \%$ y más de población con hacinamiento medio o alto por 1.000 habitantes $(\mathrm{H}=13.670$; $\mathrm{gl}=3 ; \mathrm{p}=$ 0,00335). Luego, aquellos habitantes residentes en los barrios donde los tres factores de riesgo están presentes tuvieron un riesgo relativo de 1,3 de enfermar de tuberculosis en relación con aquellos viviendo en barrios sin ningún factor de riesgo presente.

No se demuestra la existencia de una asociación significativa entre las tasas de incidencia de los barrios ni con los niveles de educación, ni con el estado técnico de las viviendas predominante en ellos (Tabla 1).

\section{Discusión}

Los resultados de este trabajo evidencian la magnitud del problema de la incidencia de tuberculosis en el municipio Marianao. En el período 1995-2000 los habitantes del municipio Marianao presentaron un riesgo de enfermar de tuberculosis pulmonar o extrapulmonar casi 2 veces superior al de un habitante de Cuba en su totalidad y 1,5 veces mayor al de un residente de la provincia Ciudad de La Habana.

El análisis de la distribución espacial de las tasas de incidencia a nivel de barrio con la aplicación del método bayesiano empírico permitió identificar un agrupamiento de barrios con tasas altas (superiores a la media municipal) en la región central del municipio. En estos barrios el factor de riesgo que predomina es el hacinamiento, encontrándose en ellos el 90\% o más de población en condiciones de hacinamiento medio o alto.

Como en otros estudios en los que se analizan áreas de grupos poblacionales (Vieira, 2000,2001 ), se ha obtenido una diferenciación espacial de la ocurrencia de tuberculosis en un municipio urbano que revela las zonas que presentan mayor necesidad de intervención.

Los factores de riesgo identificados asociados a la incidencia de tuberculosis son: el porcentaje de familias con problemas disfuncionales en el orden socioeconómico, psicológico y/o de higiene ambiental, el porcentaje de población con diversos niveles de hacinamiento, y la densidad poblacional. Con respecto a la relación inversa entre la densidad poblacional y la incidencia de tuberculosis, recomendamos continuar analizando el problema en posteriores estudios.

La incidencia de tuberculosis en el municipio Marianao no está asociada ni con los niveles de educación, ni con el estado de la vivienda predominante en el barrio. En Cuba la población de manera general presenta un alto nivel educacional, debido a los esfuerzos del go- 
Asociación entre las tasas de incidencia de tuberculosis de los barrios con el nivel de educación

y estado técnico de las viviendas.

\begin{tabular}{|c|c|c|}
\hline Análisis & Método & Resultados \\
\hline $\begin{array}{l}\text { Clasificación de los barrios } \\
\text { según tasa (alta o no) y nivel } \\
\text { de educación predominante }\end{array}$ & Prueba $X^{2}$ de asociación & $\begin{array}{l}X^{2} \text { Yates }=0,122 \\
p=0,07268\end{array}$ \\
\hline $\begin{array}{l}\text { Diferencias de tasas para todo } \\
\text { nivel de educación predominante. }\end{array}$ & Prueba de Kruskal-Wallis & $\begin{array}{l}H=0,879, g l=2 \\
p=0,6442\end{array}$ \\
\hline $\begin{array}{l}\text { Diferencias de tasas para distintas } \\
\text { agrupaciones de categorías de nivel } \\
\text { de agrupación predominante. }\end{array}$ & $\begin{array}{l}\text { Prueba de Wilcoxon-Mann-Whitney } \\
\text { (Primaria vs. Secundaria + Preuniversitario) } \\
\text { (Primaria + Secundaria vs. Preuniversitario) }\end{array}$ & $\begin{array}{l}z=-0,859 ; p=0,1951 \\
z=0,204 ; p=0,4192\end{array}$ \\
\hline $\begin{array}{l}\text { Clasificación de los barrios según } \\
\text { su tasa (alta o no) y nivel de estado } \\
\text { de la vivienda predominante. }\end{array}$ & Prueba $X^{2}$ de asociación & $\begin{array}{l}X^{2}{ }_{\text {Yates }}=0,122 \\
p=0,7268\end{array}$ \\
\hline $\begin{array}{l}\text { Diferencia de tasas para agrupaciones } \\
\text { de niveles de estado de vivienda } \\
\text { predominante. }\end{array}$ & $\begin{array}{l}\text { Dócima de Wilcoxon-Mann-Whitney } \\
\text { (Bueno vs. Regular + Malo) }\end{array}$ & $z=-0,102 ; p=0,4594$ \\
\hline
\end{tabular}

bierno por la educación del pueblo. Con respecto al estado de la vivienda, según informes realizados por la Dirección Municipal de Planificación Física (Mondéjar, 1999) el municipio presenta sólo el $6,4 \%$ del total de sus viviendas en mal estado.

Este trabajo propone la utilización del enfoque ecológico al análisis de la tuberculosis rom- piendo límites político-administrativos para identificar zonas donde residen grupos poblacionales de alto riesgo para contribuir a la planificación de acciones diferenciadas en el interior de los territorios y a la estrategia del Programa Nacional de Control de la eliminación de esta enfermedad en Cuba.

\section{Agradecimientos}

Este trabajo fue realizado en el marco del Proyecto: Evaluación de estrategias para la localización de casos de tuberculosis en municipios seleccionados de la Ciudad de La Habana por investigadores del Instituto de Medicina Tropical "Pedro Kourí" y la Dirección Municipal de Salud de Marianao.

\section{Referencias}

ALVAREZ-SINTES, R., 2001. Temas de Medicina General Integral. v. I. La Habana: Editorial Ciencias Médicas.

BAILEY, T. C., 2001. Spatial statistical methods in health. Cadernos de Saúde Pública, 17:1083-1098.

BISHAI, W. R.; GREHAM, N. M.; HARRINGTON, S.; POPE, D. S.; HOOPER, N.; ASTEMBORSKI, J.; SHEELY, L.; VLAHOV, D.; GLASS, G. E. \& CHAISSON, R. E., 1998. Molecular and geographic patterns of tuberculosis transmission after 15 years of directly observed therapy. JAMA, 280:16791684.

BRADLEY, J. V., 1968. Distribution-free Statistical Tests. New Jersey: Prentice-Hall.

CÂMARA, G. \& MONTEIRO, A. M. V., 2001.Geocomputation: techniques for spatial analysis: Are they relevant to health data? Cadernos de Saúde Pública, 17: 1059-1071. 
FONSECA-NOBRE, F. \& CARVALHO, M. S., 1996. Spatial and Temporal Analysis of Epidemiological Data. May 1999 <http://www.idrc.ca/books/focus/ 766/nobre.html>.

INSTITUTO NACIONAL DE LA VIVIENDA, 1990. Procedimiento para Determinar el Estado Técnico de la Vivienda. La Habana: Instituto Nacional de la Vivienda.

KANJI, G. K., 1993. 100 Statistical Tests. London: Sage Publications.

KUNST, A. E. \& MACKENBACH, J. P., s. d. Measuring Socioeconomic Inequalities in Health. Copenhagen: World Health Organization.

MARTINS, R., 1998. Mapas de taxas epidemiológicas: Uma abordagem Bayesiana. Cadernos de Saúde Pública, 14:713-723.

MINSAP (Ministerio de Salud Pública), 1998. Salud en el Tiempo. La Habana: MINSAP.

MINSAP (Ministerio de Salud Pública), 1999a. Dirección Nacional de Epidemiología. Programa Nacional de Control de Tuberculosis: Manual de Normas y Procedimientos. La Habana: Editorial Ciencias Médicas.

MINSAP (Ministerio de Salud Pública), 1999b. Anuario Estadístico 1998. La Habana: MINSAP.

MINSAP (Ministerio de Salud Pública), 2000. Anuario Estadístico 1999. La Habana: MINSAP.

MINSAP (Ministerio de Salud Pública), 2001. Anuario Estadístico 2000. La Habana: MINSAP.

MONDEJAR, L. E., 1999. La Vivienda en el Municipio Marianao. Diagnósticos y Retos. Informe Técnico. Marianao: Dirección Municipal de Planificación Física.
MOSTELLER, R. \& ROURKE, R. E. K., 1973. Study Statistics; Nonparametrics and Order Statistics. Boston: Addison-Wesley.

OFICINA NACIONAL DE ESTADISTICAS, 2000. Indicadores Demográficos por Provincias y Municipios. La Habana: Oficina Nacional de Estadística.

SANTOS, M., 1994. Por una Geografía Nueva. Madrid: Editorial Espasa.

SIEGEL, S., 1956. Nonparametric Statistics for the Behavioral Sciences. New York: McGraw-Hill.

SILVA, L. C., 1996. Cultura Estadística e Investigación en el Campo de la Salud; Una Mirada Crítica. Madrid: Editorial Díaz de Santos.

STEEL, R. G. D. \& TORRIE, J. H., 1960. Principles and Procedures of Statistics. New York: McGraw-Hill.

VIEIRA, W.; BARCELLOS, C.; BRITO, A. M.; CARVALHO, M. S.; GONÇALVEZ-CRUZ, O.; ALBUQUERQUE, M. F; RIBEIRO, K. \& LAPA, T. M., 2001. Aplicação de modelo bayesiano empírico na análise espacial da ocurrencia de hanseníase. Revista de Saúde Pública, 35:474-480.

VIEIRA, W.; XIMENES, R.; ALBUQUERQUE, M. F.; LAPA, T. M.; PORTUGUAL, J. L.; LIMA, M. L. C. \& MARTELLI, C. M. T., 2000. The use of socioeconomic factors in mapping tuberculosis risk areas in a city of northeastern Brazil. Panamerican Journal of Public Health, 8:403-410.

WERNECK, G. L. \& STRUCHINER, C. J., 1997. Estudos de agregados de doença no espaço-tempo: Conceitos, técnicas e desafios. Cadernos de Saúde Pública, 13:611-624.

Recibido el 6 de junio de 2002

Versión final vuelta a presentar el 17 de febrero de 2003 Aprobado el 6 de mayo de 2003 